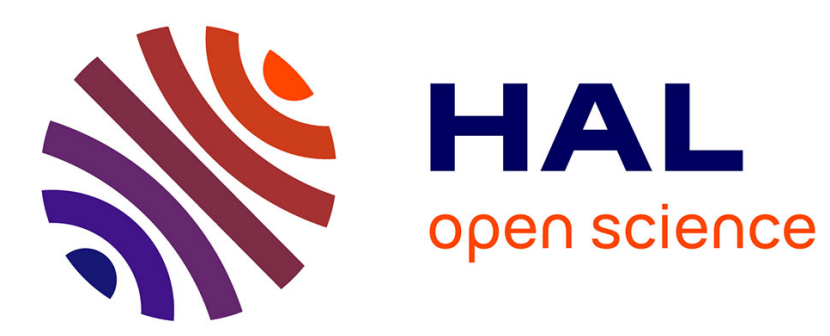

\title{
Physical investigation of the Electro-Acoustic-Reflectometry method for space charge measurements
}

\author{
L Hamidouche, E. Géron, S. Holé
}

\section{- To cite this version: \\ L Hamidouche, E. Géron, S. Holé. Physical investigation of the Electro-Acoustic-Reflectometry method for space charge measurements. Physica Scripta, 2019, 94 (11), pp.115006. 10.1088/1402- 4896/ab2105 . hal-02967707}

\section{HAL Id: hal-02967707 https: / hal.sorbonne-universite.fr/hal-02967707}

Submitted on 15 Oct 2020

HAL is a multi-disciplinary open access archive for the deposit and dissemination of scientific research documents, whether they are published or not. The documents may come from teaching and research institutions in France or abroad, or from public or private research centers.
L'archive ouverte pluridisciplinaire HAL, est destinée au dépôt et à la diffusion de documents scientifiques de niveau recherche, publiés ou non, émanant des établissements d'enseignement et de recherche français ou étrangers, des laboratoires publics ou privés. 


\title{
Physical Investigation of the Electro-Acoustic-Reflectometry Method for Space Charge Measurements
}

\author{
L. Hamidouche, E. Géron and S. Holé \\ Laboratoire de Physique et d'Étude des Matériaux (LPEM) \\ ESPCI-Paris, PSL University - Sorbonne Université - CNRS \\ 10, rue Vauquelin - 75005 Paris - France \\ stephane.hole@espci.fr
}

(Dated: 2019/04/19 (revised version))

\begin{abstract}
The Electro-Acoustic Reflectometry (EAR) method was recently proposed for the purpose of probing space charge with a high spatial resolution. In this article, the underlying physical principle of the method is studied in order to explain the observed signals. The effect of boundary conditions is exploited to experimentally validate the theoretical calculations.
\end{abstract}




\section{INTRODUCTION}

The challenge facing today the non destructive methods for space charge measurement in dielectric materials is achieving a better bulk spatial resolution than the already existing one which is about $10 \mu \mathrm{m}$ at best [1,2]. Actually, the need for enhancing the spatial resolution occurs due to the continuous miniaturization trend in technology, on one hand, and to the use of higher voltages such as in High Voltage Direct Current (HVDC) systems, becoming more common, on the other hand. In fact the higher the operating voltage or the thinner the dielectric material, the larger the electric field and the more likely is the charge injection into the dielectric materials of the electronic devices and hence the quicker the dielectric breakdown is reached.

The usual non destructive measurement methods can be grouped into three categories, the Thermal method [3-5], the Pressure-Wave-Propagation (PWP) method [6-8] and the Pulsed-Electro-Acoustic (PEA) method $[9,10]$. These methods all share the same physical working principle $[2,11]$ but differ in their implementation and their processing techniques. The main idea in the measurement of space charge with these methods is sending a physical perturbation to the sample containing space charge and measuring its response; either electric or elastic. The efforts in this area of research are now focused on improving the spatial resolution of these methods [12-15]. While the limitation of the thermal method is purely physical as it is due to the diffusion nature of the excitation $[14,16]$, it is mainly imposed by available technology for the elastic methods as it is due to insufficient bandwidth of the measurement system.

The Electro-Acoustic-Reflectometry (EAR) method has been introduced [17-19] to overcome this problem. A review of the basics of the EAR method is given in Section II. The physical model which is based on the state equations of an electro-elastic coupling is addressed in Section III. Simulation results are presented in Section IV followed by experimental results in Section $\mathrm{V}$ for validation.

\section{EAR METHOD BASICS}

When an insulating material is subjected to an electrical excitation, the variation of the electrostatic forces due to the charges inside the material gives birth to elastic waves. In 
the case of the PEA method, these waves are transmitted to a wave-guide and then to a piezoelectric transducer for measurement. High resolution requires fast rise-time excitation [2], and thus high frequency components. At high frequency, it is very hard to manufacture good elastic couplings to ensure the non dispersion of the elastic waves traveling from the sample toward the transducer, because elastic waves greatly scatter on any defect when the wavelength is very small (namely, at high frequency) which destroys the signal. With the EAR method, the electrical energy consumed for the generation of the elastic waves is measured instead of directly measuring the elastic waves. In fact, since the sample can be viewed as an electrical load, one part of the incident electrical excitation is absorbed by the sample and the other part is reflected back to the generator; the sum of the energy of these two parts being equal to the energy of the excitation. Hence, if elastic waves are generated after excitation, more energy is absorbed by the sample and thus less energy is reflected back to the generator. As a consequence, the reflected electrical signal, which links directly to the electrical impedance of the sample, holds an attribute of the elastic signal which is generated within the sample. In addition, excitation and measurement are made in frequency domain as it is more convenient at high frequencies. Figure 1 illustrates the EAR method principle.

Making the measurement frequency by frequency is equivalent to using the frequency content of a time domain impulse. In theory, a perfect impulse function has an infinite amplitude and a zero-time duration, thus infinite frequency span spectrum. In practice, the amplitude of an impulse and its duration are always finite as well as its spectrum. There is an inverse relationship between the impulse duration and its spectrum bandwidth. The shorter the time duration of the impulse, the larger its spectrum. To achieve the required spatial resolution for the measurement of space charge in thin films, the impulse duration must be as short as possible, or at least its rise time. Actually, the rise time value is directly linked to the spatial resolution with elastic measurement methods [2]. Therefore, instead of using a time domain impulse, its frequency components are used. The broader the spectrum, the shorter the equivalent time domain impulse, hence the spatial resolution can be enhanced.

It is desired to reach a bulk spatial resolution below $10 \mu \mathrm{m}$. For instance to get to $1-\mu \mathrm{m}$ spatial resolution, the excitation bandwidth must be $1 \mathrm{GHz}$ in the case of most polymers. At microwave frequencies, the conventional approach for measuring voltages and currents does not apply because of the propagation phenomenon of the electric waves that occurs whenever their wavelength is small compared to the size of the measurement circuit elements. Given 
this, a microwave setup is more suitable for the implementation of the EAR method.

In microwave theory, the sample can be considered as a black box which is fully characterized by its scattering matrix in a one-port configuration as shown in Figure 2. The scattering matrix element of interest in our case is $S_{11}$ which holds the reflected signal from the sample. It is worth noting that this parameter is dimensionless as it is the ratio of the reflected electric wave from the sample to the incident electric wave on the sample from excitation as shown in Figure 2. If the sample were purely dielectric, its impedance $Z_{d}$ would be a capacitance in parallel with a conductance whereas, in the presence of space charges, a mechanical impedance $Z_{m}$ adds in parallel to $Z_{d}$ so the overall impedance representing the sample is $Z_{s}=Z_{m} \| Z_{d}$ as described in figures $1 \mathrm{~b}$ and $1 \mathrm{~d}$, respectively.

The reflection coefficient $S_{11}$ is directly related to the sample electric impedance $Z_{s}$ and is given by

$$
S_{11}=\frac{Z_{s}-Z_{0}}{Z_{s}+Z_{0}}
$$

where $Z_{0}$ is the impedance of the line connecting the generator to the sample which is equal to $50 \Omega$ with the standard instrumentation. In the case of the electro-elastic coupling, the electric impedance of the sample $Z_{s}$ is modified due to the appearance of a motional branch of impedance $Z_{m}$ as shown in Figure 1 and the reflection coefficient varies. This clearly shows that the reflected electric signal holds information about space charges within the sample under test.

\section{PHYSICAL MODELING}

The EAR method is an all electrical-method since both the excitation and the measured signal are of electrical nature. However, it relies on an electro-elastic phenomenon which causes the generation of elastic waves inside the insulating material. Actually the electroelastic effect takes place within any insulating material whenever subjected to an electric excitation. This is described in physics by the set of state equations in which stress, deformation, electric displacement and electric field are related through the material stiffness, its permittivity and its electro-elastic coefficient. Since excitation is made at a single frequency at a time and lock-in measurement is used at the same frequency, static and higher order electro-elastic effects vanish and only linear effects remain. As a consequence the set of state equations which is the combination of Hooke's law and Maxwell's equation for the electric 
displacement could be written in one dimension as

$$
\left\{\begin{array}{l}
T(\omega)=c S(\omega)-e^{\star} E(\omega) \\
D(\omega)=\epsilon^{\star} E(\omega)+e^{\star} S(\omega)
\end{array}\right.
$$

where $\omega$ is the angular frequency at which excitation and measurement are made, $T$ is the mechanical stress, $S$ is the mechanical deformation, $D$ is the electric displacement, $E$ is the applied electric field, $c$ is the elastic stiffness, $\epsilon^{\star}$ is the equivalent permittivity and $e^{\star}$ is the equivalent piezoelectric coefficient of the material. The equivalent permittivity is $\epsilon^{\star}=\epsilon+a S_{0}$, where $\epsilon$ is the material permittivity at rest, $a$ is the electrostrictive coefficient and $S_{0}$ is the static deformation of the sample. The equivalent piezoelectric coefficient is $e^{\star}=e+(a-\epsilon) E_{0}$, where $e$ is the actual piezoelectric coefficient of the material and $E_{0}$ is the static electric field in the sample. For the sake of simplicity, the stars are dropped hereafter.

The uni-dimensional assumption (Figure 3) holds since the other dimensions of the sample are infinitely large with respect to the thickness considered in the practical case of thin films during measurement. In other words, boundary effects are negligible compared to surface effects.

The mechanical deformation is given by

$$
S(x, t)=\frac{\partial u(x, t)}{\partial x}
$$

where $u(x, t)$ is the mechanical displacement in a longitudinal mode of propagation along the $x$-axis. The electric current density $j(t)$ generated through electro-elastic coupling can be obtained by writing the Maxwell-Ampère's law assuming a uniform magnetic field within the sample. One has

$$
j(t)=-\frac{\partial D(x, t)}{\partial t}
$$

As to the propagation equation, one has for a propagation along the thickness of the sample

$$
m_{v} \frac{\partial^{2} u(x, t)}{\partial^{2} t}+c \frac{\partial^{2} u(x, t)}{\partial^{2} x}=f(x, t)
$$

where $f(x, t)$ is the force density acting on the material and $m_{v}$ is the mass density. The solution to the homogeneous equation, that is to say out of the sources, is

$$
u(x, t)=A \exp (\imath \omega t-\imath k x)+B \exp (\imath \omega t+\imath k x)
$$


where $A$ and $B$ are the amplitudes of the elastic waves propagating in the positive and negative $x$-directions, respectively, $k$ is the wave number and $\imath$ is the imaginary constant.

However, in the case where a bulk charge is present within the sample, it constitutes a source for the elastic wave and must be taken into consideration in the expression of the material displacement. In that line of thought, one has to consider the general case where a bulk charge is present within the sample and a voltage is applied to it. In this situation, image charges are induced on the electrodes due to both the bulk charge and the applied voltage. If a charge $\sigma$ is present at position $x_{s}$ inside the sample of thickness $d$ to which a voltage $V_{0}$ is applied, then charges $\sigma_{1}$ and $\sigma_{2}$ are induced on the electrodes as shown in Figure 4c. These are the superposition of the induced charges $\sigma_{1}^{\prime}$ and $\sigma_{2}^{\prime}$ by the applied voltage alone, and the induced charges $\sigma_{1}^{\prime \prime}$ and $\sigma_{2}^{\prime \prime}$ by the space charge alone. These induced charges can be written as function of the bulk charge $\sigma$ and the applied voltage $V_{0}$ as

$$
\begin{cases}\sigma_{1}=\sigma_{1}^{\prime}+\sigma_{1}^{\prime \prime}=-\frac{\epsilon V_{0}}{d}-\frac{\sigma\left(d-x_{s}\right)}{d} & \text { at } x=0 \\ \sigma_{2}=\sigma_{2}^{\prime}+\sigma_{2}^{\prime \prime}=+\frac{\epsilon V_{0}}{d}-\frac{\sigma x_{s}}{d} & \text { at } x=d\end{cases}
$$

Including the contribution of all charges to the displacement and dropping the harmonic dependence $\exp (\imath \omega t)$ for sake of clarity, the overall mechanical displacement can be rewritten as

$$
\begin{aligned}
u(x)= & A \exp (-\imath k x)+B \exp (\imath k x) \\
& +\frac{\sigma \delta E}{2 \imath \omega Z} \exp \left(-\imath k\left|x-x_{s}\right|\right) \\
& +\frac{\sigma_{1} \delta E}{2 \imath \omega Z} \exp (-\imath k|x|) \\
& +\frac{\sigma_{2} \delta E}{2 \imath \omega Z} \exp (-\imath k|x-d|)
\end{aligned}
$$

One has only to inject this term into the set of state equations and use the MaxwellAmpère's law to compute the generated electric current density $j(t)$. One has to bear in mind that the electric current density is related to the voltage applied at angular frequency $\omega$ as $\delta V(\omega)=Z_{s} j(\omega)$, where $Z_{s}$ is the impedance of the sample. Therefore, the information about space charge held in the change of impedance of the sample can be found in the generated electric current as well. Hence, these quantities can be used interchangeably to investigate space charges.

The computed generated current density given in frequency domain is $j(\omega)=j_{d}(\omega)+$ 
$j_{m}(\omega)$ with

$$
\left\{\begin{aligned}
j_{d}(\omega) & =\imath \omega \frac{\epsilon}{d} \delta V(\omega) \\
j_{m}(\omega) & =\imath \omega \frac{\epsilon V_{0}+\sigma\left(d-x_{s}\right)}{d^{2}} u(0) \\
& +\imath \omega \frac{\sigma x_{s}-\epsilon V_{0}}{d^{2}} u(d)-\imath \omega \frac{\sigma}{d} u\left(x_{s}\right)
\end{aligned}\right.
$$

One can see clearly in the expression of the electric current density the presence of a first term $j_{d}(\omega)$ depending on the applied varying electrical voltage $\delta V(\omega)$ and 3 others in $j_{m}(\omega)$ depending on the elastic displacement $u(x)$. One can identify in $j_{d}(\omega)$ the sample admittance corresponding to a per unit surface capacitance of $\epsilon / d$. This term holds the dielectric behavior of the sample. The 3 other terms in $j_{m}(\omega)$ contain the contribution of the assumed bulk charge $\sigma$ at position $x_{s}$, the contribution of the induced charge $\sigma_{1}$ at position 0 and the contribution of the induced charge $\sigma_{2}$ at position $d$. These contributions depend on the elastic displacement at positions $x_{s}, 0$ and $d$, respectively. Hence, the information about the space charge can be recovered from the 3 last terms.

A transformation to time domain by applying an inverse Fourier Transform allows to get the time domain signal corresponding to the elastic response of the sample. As the elastic signal depends on the elastic displacement at the boundaries and at the charge position, one has to solve for the elastic displacement in order to compute the electric current density. The solution consists in finding the amplitude of the waves traveling in both directions within the sample, that is to find $A$, and $B$ as a function of position. Hence, one has to take into account the boundary conditions. Given that, four situations are considered here: (i) free boundaries, (ii) stiff boundaries, (iii) stiff/free boundaries and (iv) free/stiff boundaries. The following conditions must be fulfilled for each case:

$$
\begin{cases}u(0)=u(d)=0 & \text { Stiff boundaries } \\ T(0)=T(d)=0 & \text { Free boundaries } \\ u(0)=T(d)=0 & \text { Stiff/free boundaries } \\ u(d)=T(0)=0 & \text { Free/stiff boundaries }\end{cases}
$$

These conditions imply large reflection at both interfaces. This is a good situation for the EAR method because generating standing waves inside the sample makes it easier to distinguish in the signal the effect of elastic waves from the effect of conventional dielectric 
losses [20]. Moreover, contrarily to PWP and PEA methods for which elastic waves must enter or exit the sample to implement the measurement, elastic waves are generated and measured from inside the sample in the case of the EAR method, therefore it is better to keep elastic waves inside the sample as long as possible. Although it is easier to obtain mismatched interfaces than matched interfaces, interfaces are neither perfectly stiff nor free in the general case. However, these intermediate situations can always be described by the superposition of stiff and free conditions. Thus, studying stiff and free conditions shed light on all intermediate conditions.

Applying conditions (10) yields to the exact expression of the elastic displacement in each case and one only has to substitute it in the expression of the electric current density. Solving for $A$ and $B$ in these four situations gives for the stiff boundary conditions

$$
\left\{\begin{array}{l}
A=-\frac{\sigma_{1} \sin (k d)+\sigma \sin \left(k\left(d-x_{s}\right)\right)}{2 \imath \omega Z \sin (k d)} \delta E \\
B=-\frac{\sigma_{2} \sin (k d)+\sigma \sin \left(k x_{s}\right)}{2 \imath \omega Z \sin (k d) \exp (\imath k d)} \delta E
\end{array}\right.
$$

for the free boundary conditions

$$
\left\{\begin{array}{l}
A=\frac{\sigma_{1}(1-\cos (k d))+\sigma\left(1-\cos \left(k\left(d-x_{s}\right)\right)\right)}{2 \omega Z \sin (k d)} \delta E \\
B=\frac{\sigma_{2}(1-\cos (k d))+\sigma\left(1-\cos \left(k x_{s}\right)\right)}{2 \omega Z \sin (k d) \exp (\imath k d)} \delta E
\end{array}\right.
$$

for the stiff/free boundary conditions

$$
\left\{\begin{array}{l}
A=\frac{\sigma_{1}(1-\cos (k d))+\sigma\left(1-\cos \left(k\left(d-x_{s}\right)\right)\right)}{2 \imath \omega Z \cos (k d)} \delta E \\
B=\frac{\sigma_{2} \sin (k d)+\sigma \sin \left(k x_{s}\right)}{2 \omega Z \cos (k d) \exp (\imath k d)} \delta E
\end{array}\right.
$$

and for the free/stiff boundary conditions

$$
\left\{\begin{array}{l}
A=\frac{\sigma_{1} \sin (k d)+\sigma \sin \left(k\left(d-x_{s}\right)\right)}{2 \omega Z \cos (k d)} \delta E \\
B=\frac{\sigma_{2}(1-\cos (k d))+\sigma\left(1-\cos \left(k x_{s}\right)\right)}{2 \imath \omega Z \cos (k d) \exp (\imath k d)} \delta E
\end{array}\right.
$$

\section{SIMULATION RESULTS}

The information about space charge is within the elastic component $j_{m}(\omega)$ of the generated electric current density by electro-elastic coupling. Therefore, the time domain signal is 
computed hereafter using this component in order to investigate its space charge information content.

Values of $x_{s}$ ranging from $d / 10$ to $9 d / 10$ with a step of $d / 10$ for all the four boundary conditions are investigated. The time domain signal is obtained through an inverse Fourier transform from the calculation of $j_{m}(\omega)$ by taking $\omega$ ranging from 0 to $50 \pi v_{s} / d$, where $v_{s}$ is the velocity of sound inside the sample. This corresponds to up to 50 harmonics inside the sample. In the presented figures, the spectra are plotted in a log-scale to better see the variations. These spectra correspond to the maximum resonance occurring when the denominator of the elastic wave amplitudes $A$ and $B$ approaches 0 . Slight losses are introduced in the wave number $k$ to overcome calculation divergence and sampling issues.

In all time domain figures presented hereafter, the abscissa is mapped to position within the sample using $x=v_{s} t$. As obtained signals are periodic, only the first period, which corresponds to the sample thickness $d$, and half of the second period are shown.

The computed elastic spectra for $x_{s}=3 d / 10$ with the four boundary conditions are shown in Figure 5 in the case of $\epsilon V_{0} / d=1 \mathrm{C} / \mathrm{m}^{2}$ and $\sigma=0 \mathrm{C} / \mathrm{m}^{2}$ (no internal charge). The corresponding time domain signals are shown in Figure 6. One can see from this set of results that when the boundaries are stiff and no space charge is within the sample, no signal is detected meaning the induced charges on electrodes cannot be detected. In this case, charges are bound because of the stiffness of the boundaries and cannot move in response to the excitation, hence no elastic signal is generated. When the boundaries are free, the charges induced on the electrodes by the applied voltage are well detected and in the case of stiff/free and free/stiff boundaries, only the charges on the free boundary are detected.

When no voltage is applied and a space charge is present within the sample $\left(\epsilon V_{0} / d=\right.$ $0 \mathrm{C} / \mathrm{m}^{2}$ and $\sigma=1 \mathrm{C} / \mathrm{m}^{2}$ ), an elastic signal is detected with all types of boundaries as shown in Figure 7. The observed peaks in the time domain signal correspond to the instants when a charge is displaced by the elastic waves. One should notice that this signal has not only information about the space charge but also exhibits features corresponding the signal generated by the image charges $\sigma_{1}$ and $\sigma_{2}$ as expressed in (7).

For the case of an assumed bulk charge and an applied voltage $\left(\epsilon V_{0} / d=1 \mathrm{C} / \mathrm{m}^{2}\right.$ and $\sigma=1 \mathrm{C} / \mathrm{m}^{2}$ ), one can see from Figure 8 that for all boundary types there is no major change in the time signal compared to the former case, except for the modification of the 
amplitude. Actually, there is a superposition of the two previous cases which makes this change in amplitude but without affecting the information about the charge position. This also shows that the effect of voltage alone can be deduced by combining a measurement under voltage and a measurement under short-circuit. This is very useful for making the calibration since, knowing the capacitance of the sample, it is possible to attribute to the peak amplitude in the signal the known charge quantity on the electrodes.

To gain an insight into the meaning of the obtained signals, a time-position map is used to follow the path of the generated elastic waves. This is shown for the cases of stiff boundaries and free boundaries in figures 9 and 10, respectively. The elastic waves are represented by bold arrows. Circles in the time-position map correspond to a signal generation by charge movement due to the elastic waves, with the same assumption of a bulk charge $\sigma$ at $3 d / 10$ and two charges $\sigma_{1}$ and $\sigma_{2}$ on the boundaries.

For the stiff boundary case, when the electrical excitation is applied to the sample, only the internal charge is free to move and generates elastic waves. These elastic waves have two components traveling in opposite directions along the thickness of the sample. When these components arrive at the boundaries, they bounce back in the opposite direction and continue to travel and bounce back and forth in a periodic pattern. Each time these elastic waves encounter the internal charge (see the circles), this latter is displaced and an electrical signal is generated.

In the case of free boundaries, all charges (in the bulk and on the boundaries) are free to move upon electrical excitation and all of them generate elastic waves. These waves travel along the thickness of the sample and bounce back and forth when reaching the boundaries. In this case, an electrical signal is generated whenever an elastic wave encounters a charge whether in the bulk or on the boundaries (see the circles). When more than one charge is moved at a time, all contributions sum up to form the obtained signal.

Though the signal seems to contain many spurious contributions, these contributions are not distributed at random and their amplitude is actually informative. For instance the amplitude of the 3 peaks inside the sample in the situation shown in Figure 10 are respectively proportional to $\alpha_{1}=-2 \sigma_{1} \sigma$ (at $0.3 d$ ), $\alpha_{2}=-\frac{1}{2} \sigma^{2}$ (at $0.6 d$ ) and $\alpha_{3}=-2 \sigma_{2} \sigma$ $($ at $0.7 d)$. Therefore one has in that case $\sigma=\sqrt{2 \alpha_{2}}, \sigma_{1}=\alpha_{1} / \sqrt{8 \alpha_{2}}$ and $\sigma_{2}=\alpha_{3} / \sqrt{8 \alpha_{2}}$.

One can further verify the correlation between the assumed charge position and the obtained time signal by comparing the obtained time signal for different positions of the 
bulk charge. This is shown for the four types of boundaries in figures 11 to 13. One can see from these figures that each position of the bulk charge can be traced from the time signals as each time signal is specific to the bulk charge position. For sake of clarity, each signal is shifted upward depending on the position of the bulk charge.

\section{EXPERIMENTAL RESULTS}

The measurement setup is shown in Figure 15. It comprises a two-port vector network analyzer (VNA, HP8722ES) and a sample holder. Only one port of the VNA is used for the experiments. Data acquisition and processing are performed with Matlab software.

To demonstrate the effect of the interface type on the measured signal, experiments were carried out using a 2-mm-diameter circular PVDF sample with two types of boundaries. First, the case of free boundaries is considered as described by the configuration in Figure 16a where the sample is put directly (dry contact) on the ground plane of the sample holder. Second, silicone oil is spread in a thin layer on the ground plane before putting the sample on top of it as illustrated in Figure 16b. Attention is paid when handling the oil so that it does not overflow on top of the sample in order to keep a free boundary from the probe side. One should note that in this situation the boundary is not perfectly stiff but is much stiffer than when using a dry contact.

The reflection coefficient $S_{11}$ is measured with the same measurement setup and the time domain signal is computed with the usual way [19]. The results are shown in Figure 17. One can see a clear reduction of the second peak of the time signal resulting with the configuration shown in Figure 16b in comparison to the resulting signal with the configuration shown in Figure 16a. This result correlates well with the expected signal from simulation results of free/stiff boundary case though the second peak does not completely vanish as in theory because in practice this boundary is not perfectly stiff.

One can further check these results by computing the elastic reflection coefficient from the signals. Indeed, the amplitude of the signal at the back interface is proportional to total displacement of the interface (see equation (9)) thus to the sum of the effects due to the incident elastic wave and to the reflected elastic wave. Therefore if $\Gamma$ corresponds to the refection coefficient at the back interface, then the amplitude of the signal is proportional to $1-\Gamma, 1$ standing from the incident wave and $-\Gamma$ standing for the fraction of the incident 
wave reflected in the opposite direction. As a consequence, the ratio of the signal amplitudes between the situations described in Figure 16a and Figure 16b depends only on the reflection coefficients $\Gamma_{a}$ and $\Gamma_{b}$ in the two situations as $\left(1-\Gamma_{b}\right) /\left(1-\Gamma_{a}\right)$. In addition, one has a total reflection in the situation described in Figure 16a, hence $\Gamma_{a}=-1$. One finds from Figure 17 a ratio of $0.65=\left(1-\Gamma_{b}\right) / 2$, thus corresponding to a reflection coefficient $\Gamma_{b} \approx-0.3$.

The acoustic reflection coefficient depends on the acoustical impedance of materials as

$$
\Gamma=\frac{Z_{t}-Z_{i}}{Z_{t}+Z_{i}} \quad \text { thus } \quad Z_{t}=Z_{i} \frac{1+\Gamma}{1-\Gamma}
$$

where $Z_{t}$ is the acoustic impedance of the material to which the wave is transmitted and $Z_{i}$ the acoustic impedance of the material holding the incident wave. It can be recalled that the acoustic impedance is the product of the velocity of sound $v_{s}$ multiplied by the mass density $m_{v}$ and is expressed in Rayl which is equivalent to kilogram per square meter per second. Knowing the acoustic impedance of the sample $Z_{i} \approx 3$ MRayl, one finds from $\Gamma_{b}$ and equation (15) an acoustic impedance $Z_{t} \approx 1.6 \mathrm{MRayl}$. This acoustic impedance is smaller than the one of Brass $\left(Z_{\text {brass }} \approx 40.4\right.$ MRayl) but very close to the one of oil ( $Z_{\text {oil }} \approx 1.4$ MRayl). In fact, it is very hard to make an oil layer thinner than $10 \mu \mathrm{m}$ even if contact points exist between the sample and the brass. As a consequence, in the scale of the transit time, the sample sees mainly the oil and not the brass and hence the reflection coefficient is closer to the one on oil instead of the one on brass.

\section{CONCLUSION}

The signal delivered by the EAR method has been modeled starting from the state equations. The linear form of the elasto-electric coupling is considered because both excitation and measurement are made at the same frequency. It is demonstrated that the reflected electrical signal from the sample contains information on the space charge distribution within the sample and on the charges on the electrodes. Various boundary conditions have been investigated. It is found that a signal is generated whenever a charge can move thus a stiff boundary do not generate a signal since it prevents the charges on that boundary to move. Experiments carried out with a 9- $\mu$ m-thick PVDF sample under various boundary 
conditions have provided very consistent results, validating the model of the EAR method.

[1] G. Teyssedre, C. Villeneuve, P. Pons, L. Boudou, K. Makasheva, and B. Despax, "Challenges in probing space charge at sub-micrometer scale," Conference on Electrical Insulation and Dielectric Phenomena, (Montreal, QC), pp. 234-237, IEEE, 2012.

[2] S. Holé, "Resolution of direct space charge distribution measurement methods," IEEE Transactions on Dielectrics and Electrical Insulation, vol. 15, pp. 861-871.

[3] R. Collins, "Practical application of the thermal pulsing technique to the study of electrets," Journal of Applied Physics, vol. 51, pp. 2973-2986, 1980.

[4] S. Bauer, S. Bauer-Gogonea, "Current practice in space charge and polarization profile measurement using thermal techniques," IEEE Transactions on Dielectrics and Electrical Insulation, vol. 10, pp. 883-902, 2003.

[5] A. Petre, D. Marty-Dessus, L. Berquez, J. L. Franceschi, S. Agnel, P. Notingher jr , J. Castellon, N. Didon, A. Toureille, "FLIMM and TSM: Two thermal methods for space charges investigation," International Conference on Solid Dielectrics, (Toulouse, France), pp. 213-216, IEEE, 2004.

[6] J. Lewiner, "Direct determination of space charge distributions in dielectrics: The pressure wave propagation method," 3rd International Conference on Conduction and Breakdown in Solid Dielectrics, (Trondheim, Norway), pp. 548-554, IEEE, 1989.

[7] J. Lewiner, S. Holé and T. Ditchi, "Pressure wave propagation methods: a rich history and a bright future," IEEE Transactions on Dielectrics and Electrical Insulation, vol. 12, pp. 114$126,2005$.

[8] S. Holé, "Recent developments in the pressure wave propagation method," IEEE Electrical Insulation Magazine, vol. 25, pp. 7-20, 2009.

[9] T. Maeno, T. Futami, H. Kushibe, T. Takada and C. M. Cooke, "Measurement of spatial charge distribution in thick dielectrics using the pulsed electroacoustic method," IEEE Transactions Electrical Insulation, vol. 23, pp. 433-439.

[10] T. Maeno, H. Kushibe, T. Takada, and C. M. Cooke, "Pulsed electro-acoustic method for the measurement of volume charges in E-beam irradiated PMMA," Conference on Electrical Insulation and Dielectric Phenomena, (Amherst, USA), pp. 389-397, IEEE, 1985. 
[11] S. Holé, T. Ditchi and J. Lewiner, "Non-destructive methods for space charge distribution measurments: What are the differences ?," IEEE Transactions on Dielectrics and Electrical Insulation, vol. 10, pp. 670-677, 2003.

[12] K. Kumaoka, T. Kato, H. Miyake, and Y. Tanaka, "Development of space charge measurement system with high positional resolution using pulsed electro acoustic method," International Symposium on Electrical Insulating Materials, (Niigata, Japan), pp. 389-392, IEEE, 2014.

[13] K. Sonoda, K. Saito, H. Miyake, Y. Tanaka, K. Sawano, T. Maruizumi, and H. Koshiishi, "Development of semiconductor sensor as a use for pulsed electro-acoustic method," International Symposium on Electrical Insulating Materials, (Toyohashi, Japan), pp. 601-604, IEEE.

[14] C. Filloy-Corbrion, S. Holé, P. Notingher, S. Agnel, L. Berquez, and D. Marty-Dessus, "Simultaneous measurement of surface temperature and space charge distribution with thermal pulse method," International Conference on Dielectrics, (Montpellier, France), pp. 53-54, IEEE, 2016 .

[15] C. Corbrion, S. Holé, P. Notingher, S. Agnel, L. Berquez, and D. Marty-Dessus, "Temperature profile estimation for high spatial resolution space charge measurement with thermal pulse methods," International Symposium on Electrical Insulating Materials, (Toyohashi, Japan), pp. 173-175, IEEE, 2017.

[16] C. Filloy-Corbrion, C. Boué, E. Géron, T. Ditchi, J. Lucas, S. Holé, P. Notingher, S. Agnel, D. Marty-Dessus, L. Berquez, and G. Teyssedre, "Surface temperature measurement for space charge distribution measurements with thermal methods," IEEE Transactions on Dielectrics and Electrical Insulation, vol. 22, pp. 1506-1511, 2015.

[17] L. Hamidouche, E. Géron, and S. Holé, "Electro-Acoustic Reflectometry, a new method toward very high spatial resolution space charge measurements," International Conference on Dielectrics, (Montpellier, France), pp. 46-48, IEEE, 2016.

[18] L. Hamidouche, E. Géron, and S. Holé, "Electro-acoustic reflectometry: Reaching high spatial resolution and high sensitivity for space charge measurements," International Symposium on Electrical Insulating Materials, (Toyohashi, Japan), pp. 219-222, IEEE.

[19] L. Hamidouche, E. Géron, and S. Holé, "Very high spatial resolution space charge measurement using electro-acoustic reflectometry (EAR)," IEEE Electrical Insulation Magazine, vol. 33, pp. $9-16$. 
[20] L. Hamidouche, S. Holé, and E. Géron, "An Overview on the Sensitivity of Electro-AcousticReflectometry (EAR) Method," IEEJ Transactions on Fundamentals and Materials, vol. 139, pp. 99-104, 2019. 


\section{LIST OF FIGURES}

1 Measurement principle of the EAR method. The sample is subjected to an electrical excitation at different frequencies (black arrow). The reflected electric signal (gray arrow) is measured. (a) If no charge is present within the sample under test, dielectric losses (dotted arrow) consume little of the incident energy and the reflection (gray arrow) is large. (b) Equivalent electric circuit of case (a) where the sample corresponds to an electrical impedance $Z_{d}$ which corresponds more or less to a capacitor. (c) If electric charges are present within the sample, the generation of elastic waves (dotted arcs) consumes additional energy through electro-elastic coupling, thus the reflection (gray arrow) is smaller. (d) Equivalent circuit of case (c) where the mechanical branch of impedance $Z_{m}$ is used to represent the effect of elastic wave generation.

2 Representation of a one-port network in transmission line theory. The reflection coefficient $S_{11}$ is given by the ratio of the reflected wave $b_{1}$ by the incident wave $a_{1}$

3 Unidimensional assumption. The lateral faces of the sample are very large

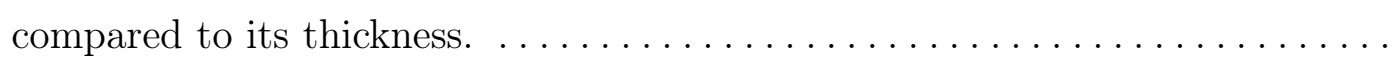

4 Different situations of induced image charges on electrodes. (a) By the applied voltage $V_{0}$. (b) By the space charge $\sigma$ at position $x_{s}$. (c) By both the space charge and the applied voltage.

5 Spectra of the elastic response at full resonance for a sample free of charge subjected to a voltage $V_{0}$. (a) Stiff boundaries. (b) Free boundaries. (c) Stiff/free boundaries. (d) Free/stiff boundaries. . . . . . . . . . . . . . .

6 Obtained signals for the four types of boundaries when no internal charge is present within the sample and a voltage is applied. (a) Stiff boundaries. (b) Free boundaries. (c) Stiff/free boundaries. (d) Free/stiff boundaries. The gray area corresponds to the sample. 
$7 \quad$ Obtained signals for the four types of boundaries when a bulk charge is present at position $x_{s}=3 d / 10$ inside the sample and no voltage is applied. (a) Stiff boundaries. (b) Free boundaries. (c) Stiff/free boundaries. (d) Free/stiff boundaries. The gray area corresponds to the sample.

8 Obtained signals for the four types of boundaries when a bulk charge is present at position $x_{s}=3 d / 10$ inside the sample and a voltage is applied. (a) Stiff boundaries. (b) Free boundaries. (c) Stiff/free boundaries. (d) Free/stiff boundaries. The gray area corresponds to the sample. ..............

9 Signal (top) and time-position map (bottom) in the case of stiff boundaries. The position of charges is indicated on the vertical axis of the time-position map. Path of the elastic waves within the sample are illustrated by bold arrows. Circles represent the instants when an elastic wave moves a charge and then generates a contribution to the signal. The gray area corresponds to the sample.

10 Signal (top) and time-position map (bottom) in the case of free boundaries. The position of charges is indicated on the vertical axis of the time-position map. Path of the elastic waves within the sample are illustrated by bold arrows. Circles represent the instants when an elastic wave moves a charge and then generates a contribution to the signal. The gray area corresponds to the sample.

11 Obtained signals with stiff boundaries for a bulk charge position from $d / 10$ to $9 d / 10$. The gray area corresponds to the sample.

12 Obtained signal with free boundaries for a bulk charge position from $d / 10$ to $9 d / 10$. The gray area corresponds to the sample. . . . . . . . . . .

13 Obtained signal with stiff/free boundaries for a bulk charge position from $d / 10$ to $9 d / 10$. The gray area corresponds to the sample.

14 Obtained signal with free/stiff boundaries for a bulk charge position from $d / 10$ to $9 d / 10$. The gray area corresponds to the sample.

15 Schematic of the measurement setup of the EAR method composed of a vector network analyzer (VNA), a computer and a sample holder. . . . . . . . . .

16 Test configurations. (a) Free boundary condition. (b) Almost free/stiff

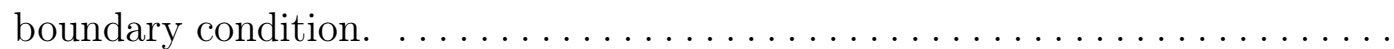


17 Obtained time domain signal with the two configurations described in Figure 16. The gray area corresponds to the sample. . . . . . . . . . . 35 


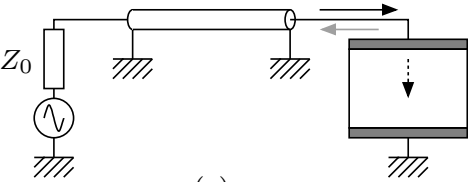

(a)

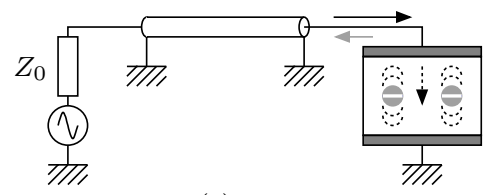

(c)

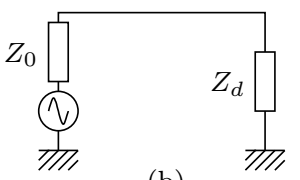

(b)

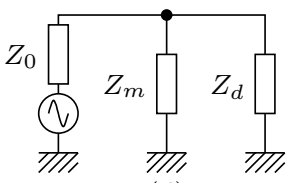

(d)

Figure 1. Measurement principle of the EAR method. The sample is subjected to an electrical excitation at different frequencies (black arrow). The reflected electric signal (gray arrow) is measured. (a) If no charge is present within the sample under test, dielectric losses (dotted arrow) consume little of the incident energy and the reflection (gray arrow) is large. (b) Equivalent electric circuit of case (a) where the sample corresponds to an electrical impedance $Z_{d}$ which corresponds more or less to a capacitor. (c) If electric charges are present within the sample, the generation of elastic waves (dotted arcs) consumes additional energy through electro-elastic coupling, thus the reflection (gray arrow) is smaller. (d) Equivalent circuit of case (c) where the mechanical branch of impedance $Z_{m}$ is used to represent the effect of elastic wave generation. 


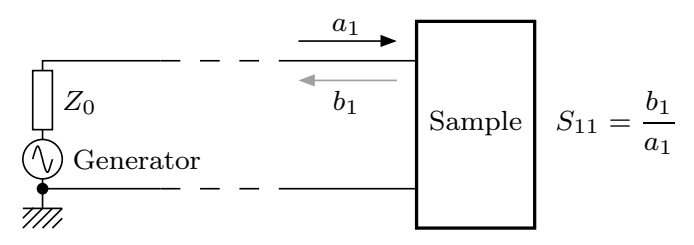

Figure 2. Representation of a one-port network in transmission line theory. The reflection coefficient $S_{11}$ is given by the ratio of the reflected wave $b_{1}$ by the incident wave $a_{1}$. 


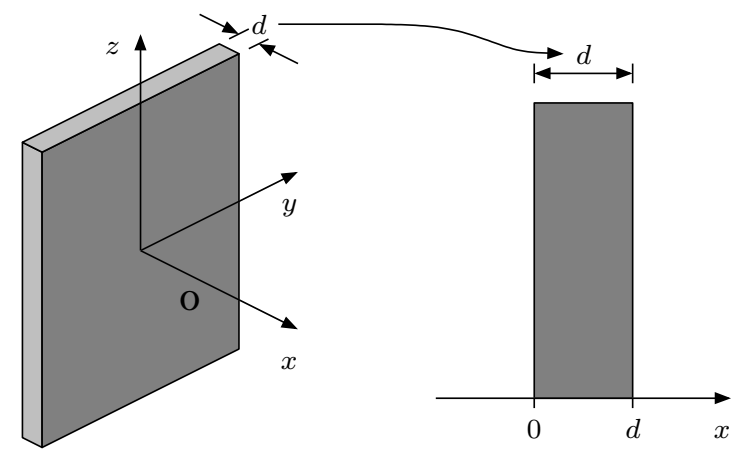

Figure 3. Unidimensional assumption. The lateral faces of the sample are very large compared to its thickness. 
(a)

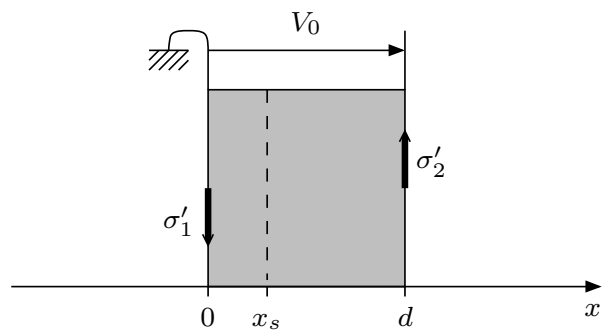

(b)

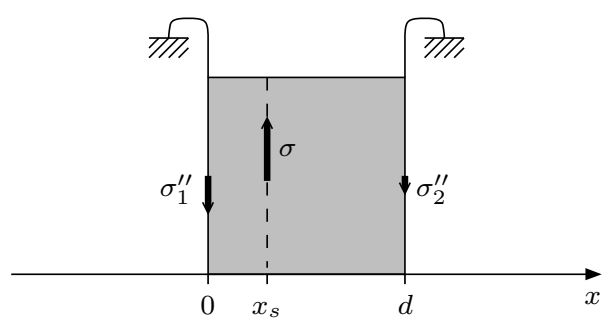

(c)

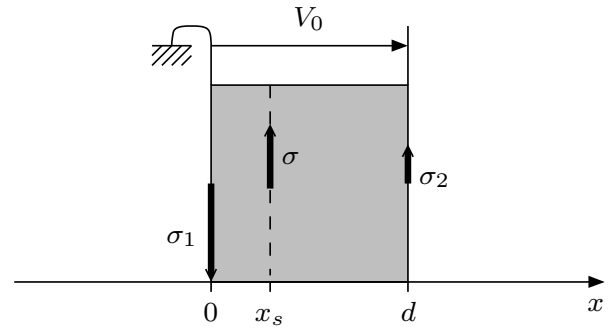

Figure 4. Different situations of induced image charges on electrodes. (a) By the applied voltage $V_{0}$. (b) By the space charge $\sigma$ at position $x_{s}$. (c) By both the space charge and the applied voltage. 
(a)

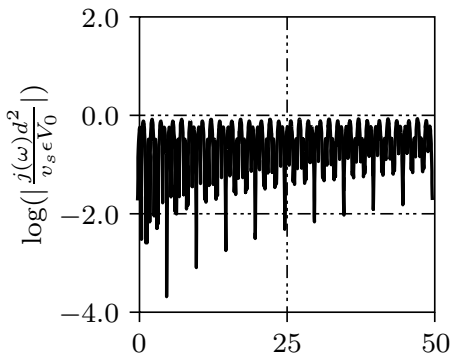

(c)

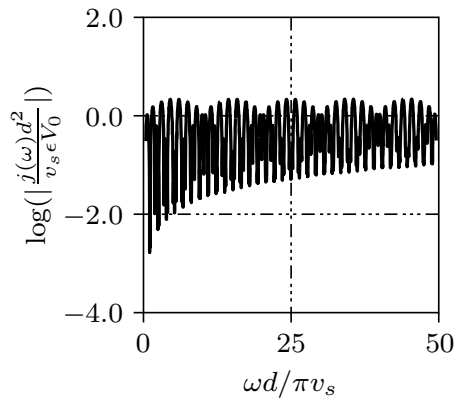

(b)

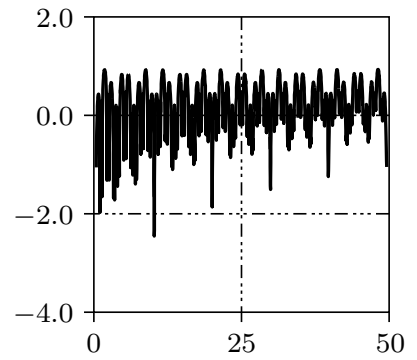

(d)

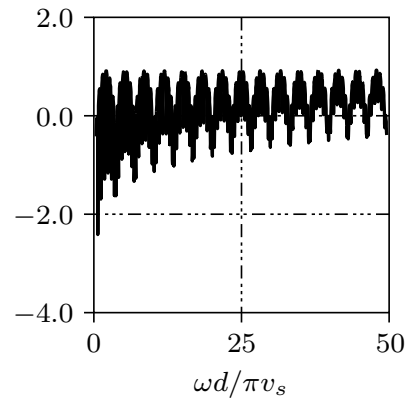

Figure 5. Spectra of the elastic response at full resonance for a sample free of charge subjected to a voltage $V_{0}$. (a) Stiff boundaries. (b) Free boundaries. (c) Stiff/free boundaries. (d) Free/stiff boundaries. 
(a)

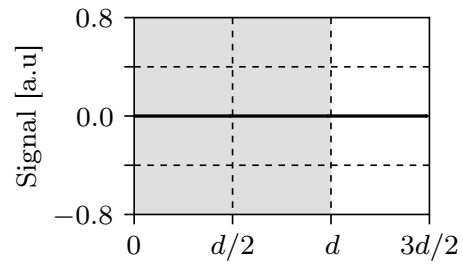

(c)

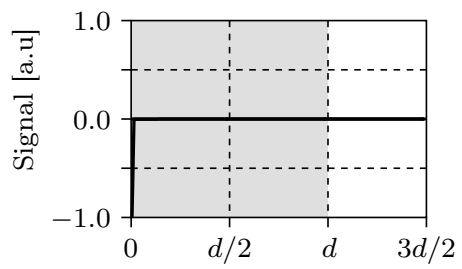

(b)

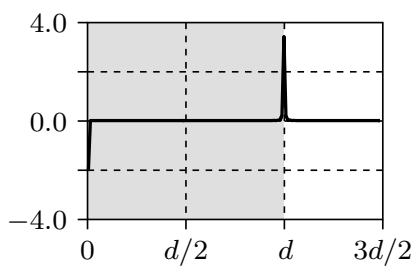

(d)

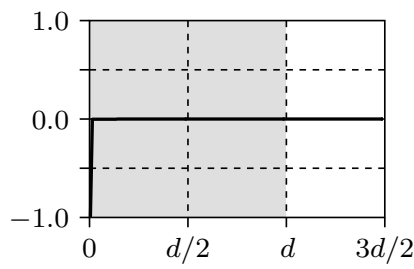

Figure 6. Obtained signals for the four types of boundaries when no internal charge is present within the sample and a voltage is applied. (a) Stiff boundaries. (b) Free boundaries. (c) Stiff/free boundaries. (d) Free/stiff boundaries. The gray area corresponds to the sample. 
(a)

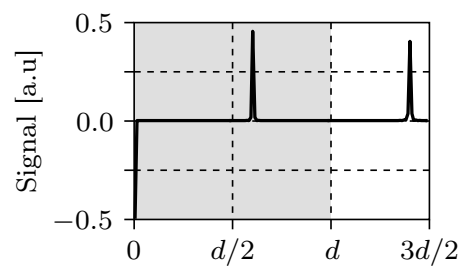

(c)

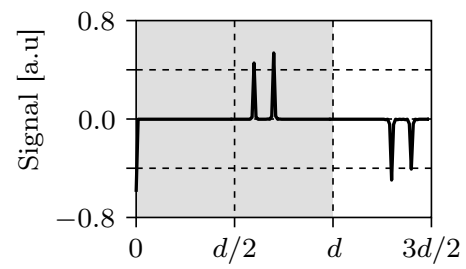

(b)

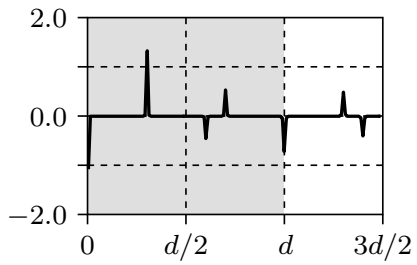

(d)

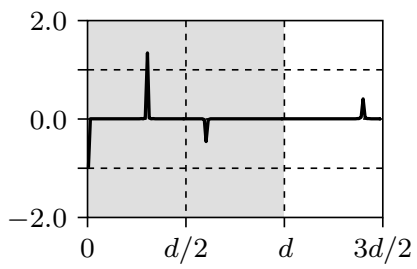

Figure 7. Obtained signals for the four types of boundaries when a bulk charge is present at position $x_{s}=3 d / 10$ inside the sample and no voltage is applied. (a) Stiff boundaries. (b) Free boundaries. (c) Stiff/free boundaries. (d) Free/stiff boundaries. The gray area corresponds to the sample. 
(a)

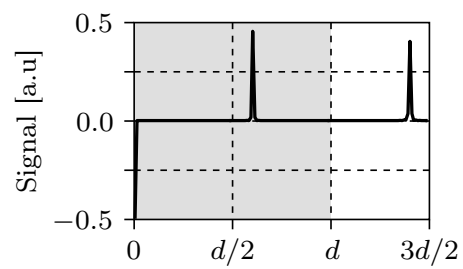

(c)

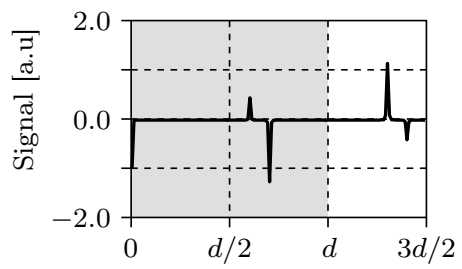

(b)

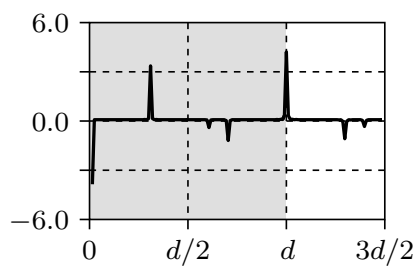

(d)

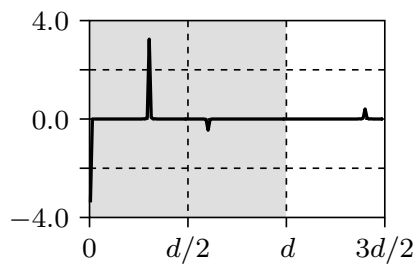

Figure 8. Obtained signals for the four types of boundaries when a bulk charge is present at position $x_{s}=3 d / 10$ inside the sample and a voltage is applied. (a) Stiff boundaries. (b) Free boundaries. (c) Stiff/free boundaries. (d) Free/stiff boundaries. The gray area corresponds to the sample. 


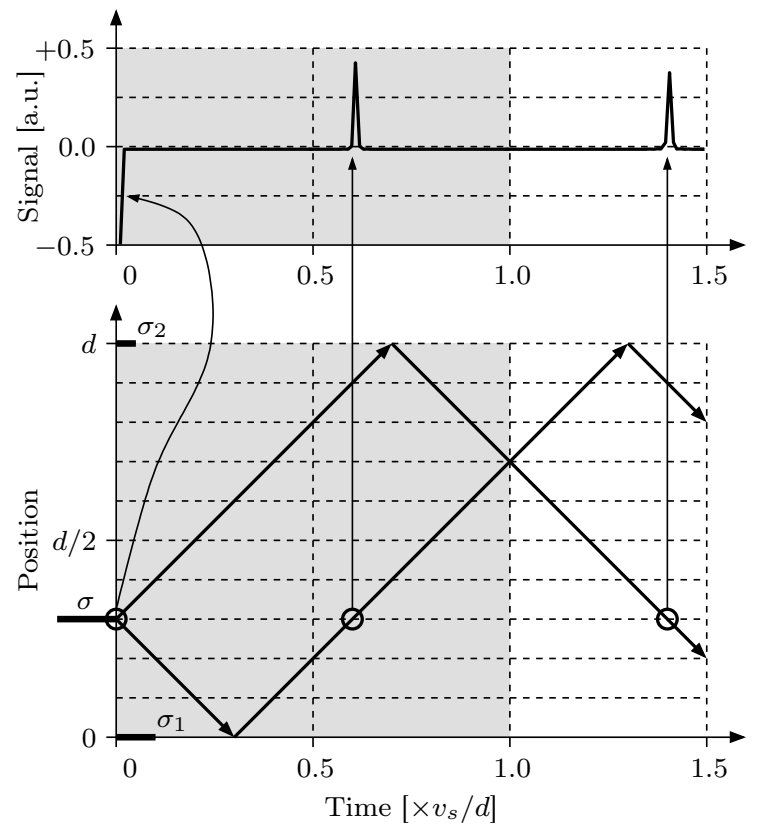

Figure 9. Signal (top) and time-position map (bottom) in the case of stiff boundaries. The position of charges is indicated on the vertical axis of the time-position map. Path of the elastic waves within the sample are illustrated by bold arrows. Circles represent the instants when an elastic wave moves a charge and then generates a contribution to the signal. The gray area corresponds to the sample. 


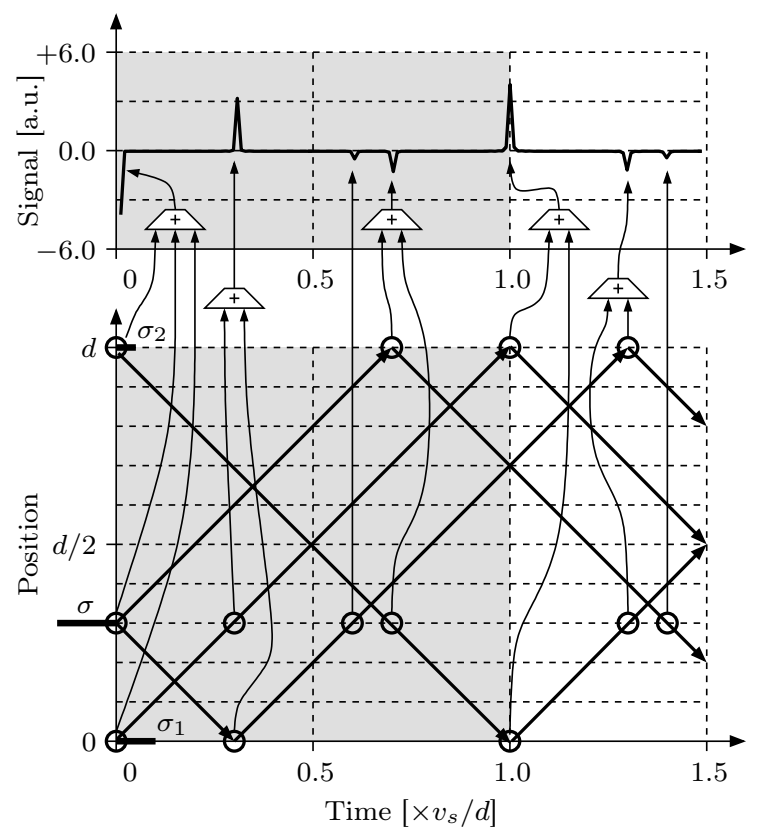

Figure 10. Signal (top) and time-position map (bottom) in the case of free boundaries. The position of charges is indicated on the vertical axis of the time-position map. Path of the elastic waves within the sample are illustrated by bold arrows. Circles represent the instants when an elastic wave moves a charge and then generates a contribution to the signal. The gray area corresponds to the sample. 


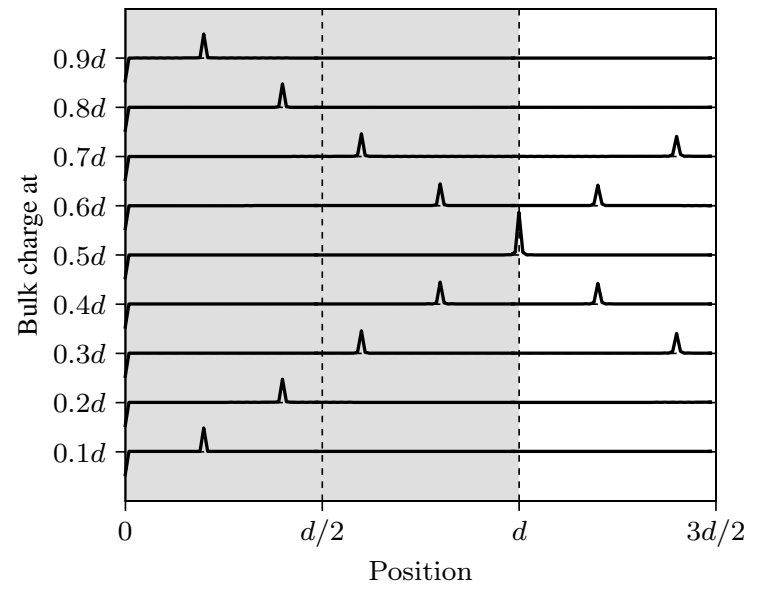

Figure 11. Obtained signals with stiff boundaries for a bulk charge position from $d / 10$ to $9 d / 10$.

The gray area corresponds to the sample. 


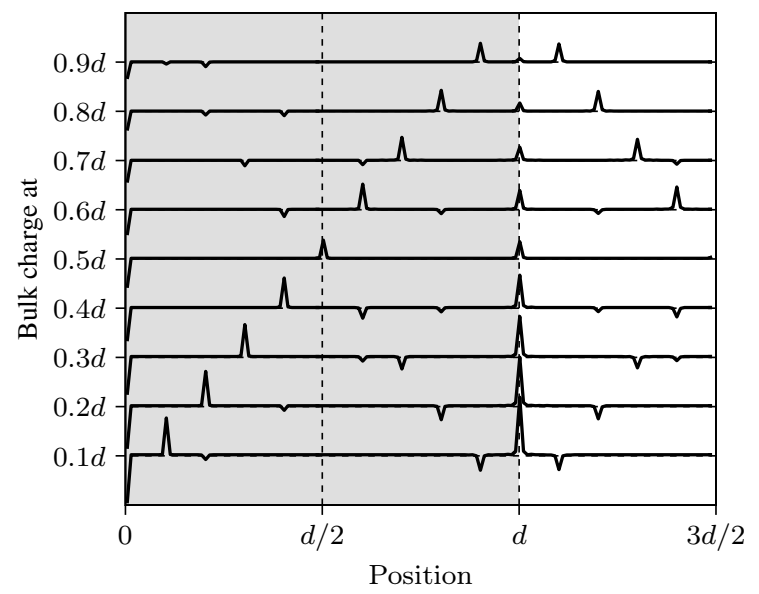

Figure 12. Obtained signal with free boundaries for a bulk charge position from $d / 10$ to $9 d / 10$. The gray area corresponds to the sample. 


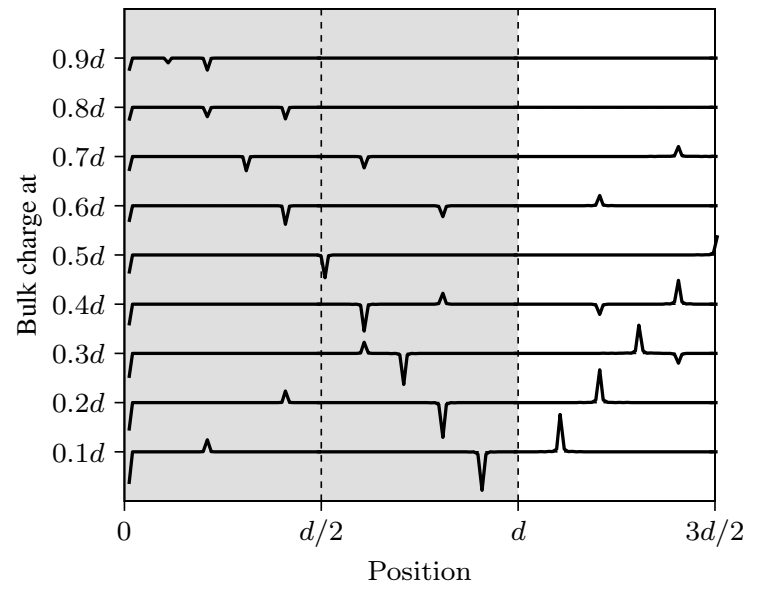

Figure 13. Obtained signal with stiff/free boundaries for a bulk charge position from $d / 10$ to $9 d / 10$.

The gray area corresponds to the sample. 


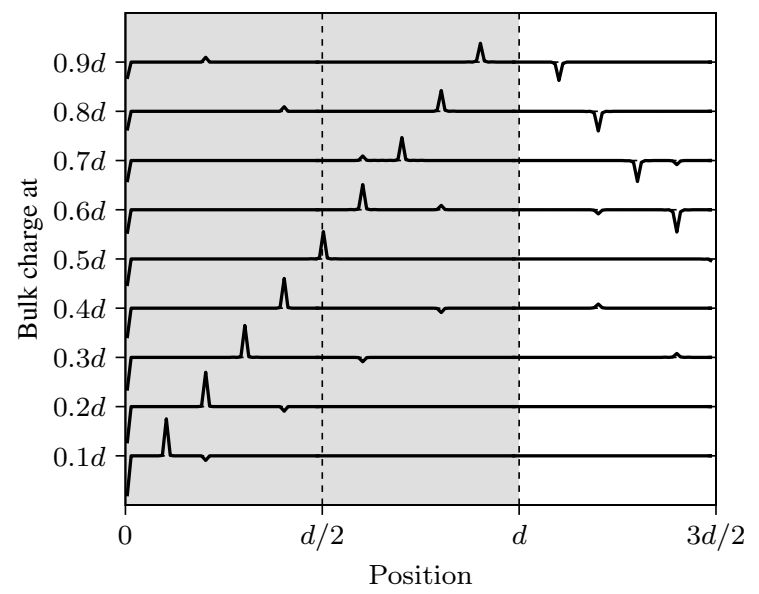

Figure 14. Obtained signal with free/stiff boundaries for a bulk charge position from $d / 10$ to $9 d / 10$.

The gray area corresponds to the sample. 


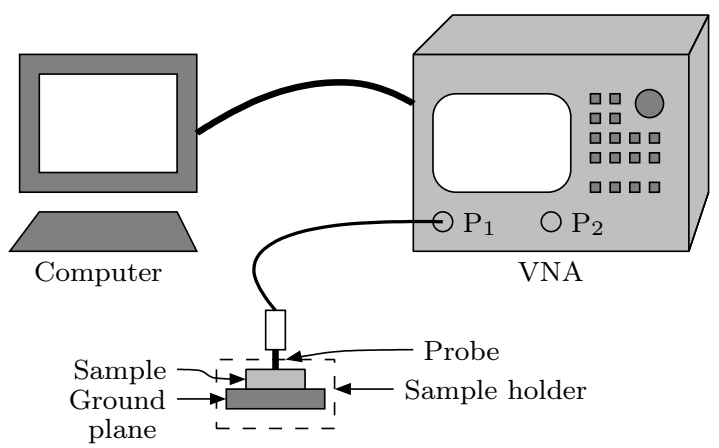

Figure 15. Schematic of the measurement setup of the EAR method composed of a vector network analyzer (VNA), a computer and a sample holder. 
(a)

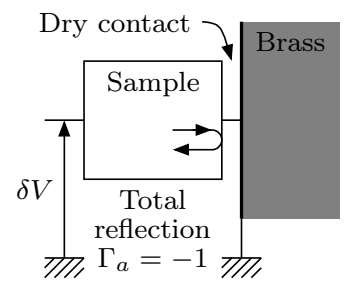

(b)

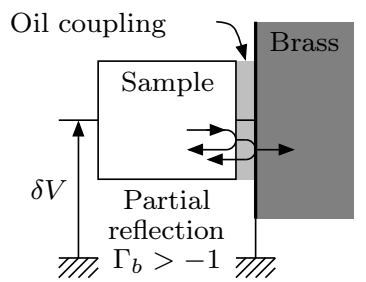

Figure 16. Test configurations. (a) Free boundary condition. (b) Almost free/stiff boundary condition. 


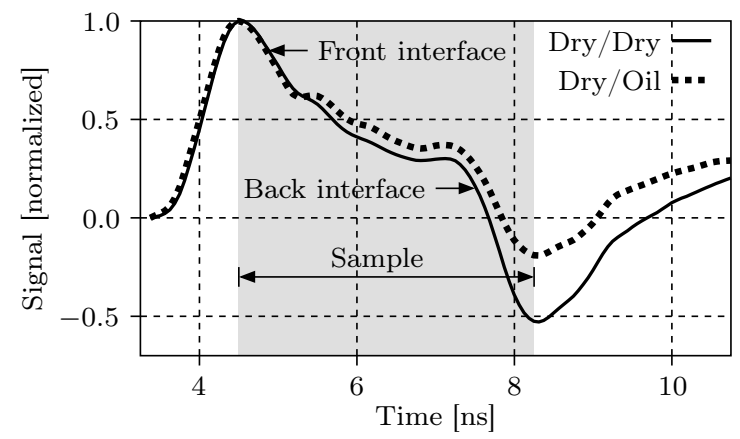

Figure 17. Obtained time domain signal with the two configurations described in Figure 16. The gray area corresponds to the sample. 